\title{
Distribution of the environmental and socioeconomic risk factors on COVID-19 death rate across continental USA: a spatial nonlinear analysis
}

\author{
Yaowen Luo ${ }^{1,2}$ (D) Jianguo Yan $^{2} \cdot$ Stephen McClure ${ }^{2}$ \\ Received: 31 July 2020 / Accepted: 21 September 2020 / Published online: 1 October 2020 \\ (C) Springer-Verlag GmbH Germany, part of Springer Nature 2020
}

\begin{abstract}
The COVID-19 outbreak has become a global pandemic. The spatial variation in the environmental, health, socioeconomic, and demographic risk factors of COVID-19 death rate is not well understood. Global models and local linear models were used to estimate the impact of risk factors of the COVID-19, but these do not account for the nonlinear relationships between the risk factors and the COVID-19 death rate at various geographical locations. We proposed a local nonlinear nonparametric regression model named geographically weighted random forest (GW-RF) to estimate the nonlinear relationship between COVID-19 death rate and 47 risk factors derived from the US Environmental Protection Agency, National Center for Environmental Information, Centers for Disease Control and the US census. The COVID-19 data were employed to a global regression model random forest (RF) and a local model GW-RF. The adjusted $R^{2}$ of the RF is 0.69 . The adjusted $R^{2}$ of the proposed GW-RF is 0.78 . The result of GW-RF showed that the risk factors (i.e. going to work by walking, airborne benzene concentration, householder with a mortgage, unemployment, airborne $\mathrm{PM}_{2.5}$ concentration and per cent of the black or African American) have a high correlation with the spatial distribution of the COVID-19 death rate, and these key factors driven from the GW-RF were mapped, which could provide useful implications for controlling the spread of the COVID-19 pandemic.
\end{abstract}

Keywords COVID-19 death rate $\cdot$ Environment $\cdot$ Socioeconomic $\cdot$ Health $\cdot$ Local nonlinear model $\cdot$ Spatial variation

\section{Instruction}

The 2019 novel coronavirus disease (COVID-19) caused by SARS-CoV-2 is a rapidly spreading infectious disease that mainly affects the respiratory system (Landi 2020). Because the disease is highly contagious with rapid transmission between humans (Huang et al. 2020), the World Health Origination (WHO) declared on March 11, 2020 that the COVID-19 outbreak is a global pandemic (World Health

Responsible editor: Lotfi Aleya

Yaowen Luo

luoyw@1reis.ac.cn

Jianguo Yan

jgyan@whu.edu.cn

1 Electronic Information School, Wuhan University, 127 Luoyu Road, Wuhan 430070, Hubei, China

2 State Key Laboratory of Information Engineering in Surveying, Mapping and Remote Sensing, Wuhan University, Wuhan, China
Organization 2020). As of July 6, 2020, a total of 11,520,953 COVID-19 confirmed cases and 532,633 deaths have been recorded worldwide. The current epicentre of the COVID-19 is the USA with 2,982,928 confirmed cases and 132,569 deaths as of July 6, 2020. The economic impact of the COVID-19 crisis is unprecedented in USA with a substantial stock market shifting and unemployment rate reaching the peak (O'Connor et al. 2020). The health care system is also overwhelmed across the world, which are already operating at full capacity struggling to meet the demand for ventilators, intensive care beds and personal protective equipment.

Some researches about the COVID-19 have found that various factors including environment (Xu et al. 2020; Ahmadi et al. 2020; Bashir et al. 2020), socioeconomic (de LeónMartínez et al. 2020; Zheng et al. 2020), demographic (Serge et al. 2020) and underlying disease (Marhl et al. 2020; Ruthberg et al. 2020; Dariya and Nagaraju 2020; Malik et al. 2020) may influence the transmission of COVID-19. Bashir et al. (2020) found that air pollution including $\mathrm{PM}_{10}, \mathrm{PM}_{2.5}, \mathrm{SO}_{2}, \mathrm{NO}_{2}$ and $\mathrm{CO}$ is a significant risk factor to the COVID-19 epidemic. Tosepu et al. (2020) 
analysed the correlation between weather and the COVID-19 and found that the average temperature was highly correlated with the COVID-19. Virus carried via public transportation played an important role in the transmission of COVID-19 (Zheng et al. 2020). Serge et al. (2020) found that males are about $60 \%$ more likely than females to suffer severe illness or death from the COVID-19 complications. Targher et al. (2020) found that patients with diabetes were at an approximately 4 times risk of having severe COVID-19. Chronic diseases such as diabetes, hypertension and cholesterol levels are apparently related to the severity of COVID-19 (Zaki et al. 2020). The risk of COVID-19 is related to blood type, in which people with blood type A have a higher risk of COVID-19, while people with blood type O have a lower risk (Pourali et al. 2020). Low-income older people are at higher risk of COVID-19 because they are more likely to suffer from chronic diseases, loneliness, uneven diet and lack of exercise etc. (Calderón-Larrañaga et al. 2020). The epidemic had a greater psychological impact on people with female gender, student status and specific diseases (e.g. hypertension and chronic lung diseases) (Wang et al. 2020).

With the increased availability of health care data online and the development of spatial analysis techniques, multiple analyses by the GIS tool (Guliyev 2020; Rosenkrantz et al. 2020) found that the distribution of COVID-19 cases (Desjardins et al. 2020; Shim et al. 2020; Lau et al. 2020) and its risk factors (Mollalo et al. 2020) exhibits patterns of spatial heterogeneity. A study by Lau et al. (2020) showed that the number of flight routes was a highly relevant factor of the COVID-19 spread. Their study showed that regions in Asia, North America and Europe were at a serious risk of constant exposure to highly infected countries, while the exposure risk to COVID-19 was relatively low in South America and Africa. Liu et al. (2020) employed a contact model to reconstruct the contact and air spread to simulate the outbreak of COVID-19 on the "diamond princess." They suggested rigorous prevention measure should be followed by highrisk susceptible people. Mollalo et al. (2020) mapped the spatial variability of the relationships between COVID-19 incidence rate and income inequality, median household, the proportion of black females and proportion of nurse practitioners using multiscale geographically weighted regression (MGWR). Sun et al. (2020) used several spatial models including spatial lag, spatial error and spatial autoregressive model to examine geographic differences in COVID-19 in US counties and found that the spatial model was able to better estimate COVID-19 prevalence in counties compared with aspatial models. Sannigrahi et al. (2020) found that the uneven distribution of the COVID19 confirmed cases and deaths across Europe, and this can be attributed to the discrepant sociodemographic factors such as the old population and income between European counties.

Many mathematical models have been employed to explore the risk factors of COVID-19. Typical global models such as partial correlation coefficient (PCC) (Ahmadi et al. 2020), ordinary least squares (OLS), Poisson regression model (Xu et al. 2020) and Bayesian hierarchical model (Millett et al. 2020) and geographical local model such as geographically weighted regression model (GWR) (Mollalo et al. 2020; Imran et al. 2015) were used to model the correlations between COVID-19 data and other impacting factors. However, the global model assumes the relationship between risk factors does not vary over space and is inconsistent with the imbalanced distribution of COVID-19. Although spatial error model (SEM) and spatial lag model (SLM) do consider spatial factors, they focus more on the analysis of spatial correlation and do not analyse the spatial variation of the relationships between variables in different regions from the perspective of spatial heterogeneity (Ahmadi et al. 2020). The GWR (Brunsdon 2010; Fotheringham et al. 2002; Lu et al. 2017) as a local regression model can obtain the linear relationship between variables in different locations. However, the GWR is constructed based on multiple linear regression models; thus, it is not suitable to estimate the nonlinear relationships between independent and dependent variables, and local multicollinearity exists when dealing with correlated variables (Wheeler and Tiefelsdorf 2005). The real relationship between risk factors and COVID19 is complex and is not always linear. In order to explore the spatial variation of the nonlinear relationship between multiple risk factors and COVID-19, it is necessary to deal with the nonlinear situation in a local regression model.

The uneven spatial distribution of COVID-19 is related to environmental and socioeconomic and demographic differences among counties. Analysis of the relationship between these possible risk factors (e.g. air pollution, old age, diabetes) and COVID-19 in different counties will be helpful in developing policies to prevent and control the spread of COVID-19. The relationship between risk factors and mortality is not completely linear in the real world. In this study, we proposed a local nonlinear nonparametric regression method, geographically weighted random forest (GW-RF), to evaluate the geographical difference in the relationship between COVID-19 death rate and multiple risk factors including air pollution, climate, land cover, disaster, health status, commuting to work and socioeconomic and demographic indicators at county level across the continental USA. This paper tries to explore the variation in the nonlinear relationships between multiple risk factors and COVID-19 death rate in different locations by using the GW-RF for the first time. We expect that this study can provide scientific evidence for implementing control and prevention measure in COVID-19.

\section{Materials and methods}

\section{Data and preparation}

The county-level daily COVID-19 death cases data and population data of 3108 counties of continental USA from Jan 22, 
2020 to June 26, 2020 were downloaded from the website of USA FACTS (https://usafacts.org/). The death rate at county level was calculated based on the daily COVID-19 death cases and population data. We selected 47 indicators including atmosphere, climate, land cover, disaster, health status, commuting to work and socioeconomic and demographic factors as independent variables to evaluate their correlation with the COVID-19 death rate. The indicators we selected and their meanings and sources are presented in Table 1. The shapefile of the selected 3108 counties was downloaded from geographical program of US Census Bureau (https://www. census.gov/programs-surveys/geography.html).

Due to the units of these 47 indicators are different, the indicators should be normalized before regression. The method is as follows:

$X_{k i}=\frac{X_{k i}-\bar{X}_{k}}{\sigma_{k}}(i \in 1,2, \cdots 2056 ; k \in 1,2, \cdots, 28)$

where $X_{k i}$ represents the normalized value of the $k$ th indicator in the $i$ th county, $X_{k i}$ represents the original value of the $k$ th indicator in the $i$ th county; $\bar{X}_{k}$ represents the average value of the $k$ th indicator; $\sigma_{k}$ represents the standard deviation of $k$ th index. The COVID-19 death rate and 47 indicators were joined to the county-level shapefile for further processing.

\section{Nonlinear nonparametric model}

RF

We selected the random forest (RF) machine learning method (Breiman 2001) because it is nonparametric; it can easily learn nonlinear relationships and interactions from data without explicitly modelling them. RF is an ensemble of multiple decision trees. The decision tree is a nonparametric model that does not have a fixed structure. The decision tree grows according to the complexity of the input data in the learning process. The RF works well for high-dimensional variables with a relatively small number of samples and can access variable importance (Grömping 2009). The algorithm flow of the RF is as follows:

1. The $n$ data sets $D_{1}, D_{2}, \cdots, D_{n}$ are extracted by repeatedly using the bootstrap method to randomly extract the whole dataset $D$, and the corresponding $n$ decision trees $H_{1}, H_{2}$, $\cdots, H_{n}$ are generated.

2. At each node of the decision tree, randomly select $m(m<$ $k$ ) variables from all the $k$ variables of the decision tree, and each node is split using the selected $m$ variables by the optimal segmentation method determined by a segmentation criterion.
3. The value of $m$ remains unchanged while the forest grows. Each tree grows to its largest extent without pruning until it cannot be split.

Thus, the correlation between the decision trees in the forest decreases through a random selection of variables at each node of the tree and the optimal split of each node is determined by the selected variables only, instead of all variables. Each tree can grow to its largest extent without pruning. Therefore, the algorithm can deal with excessive redundant features and avoid over fitting.

In the first step in constructing the RF, whether with or without replacement, approximately $36.8 \%$ of the data samples are not used to grow the tree; these samples are the out-ofbag (OOB) for the tree. The accuracy of the RF model can be estimated from the OOB data as presented by Eq. (2):

$$
\operatorname{MSE}=\frac{1}{N} \sum_{i=1}^{N}\left(y_{i}-\widehat{y}_{i}\right)^{2}
$$

where $N$ is the number of samples from the OBB data, $y_{i}$ is the actual value of the $i$ th sample, and $\overline{\widehat{y}}_{i}$ is the average prediction for the $i$ th sample from all trees.

The overall sum of squares (SST) and coefficient of determination $\left(R^{2}\right)$ are respectively defined in Eqs. (3) and (4):

$$
\begin{aligned}
& \mathrm{SST}=\sum_{i=1}^{N}\left(y_{i}-\bar{y}\right)^{2} \\
& R^{2}=1-N \frac{M S E}{S S T}
\end{aligned}
$$

where $R^{2} \in(0,1)$. The closer the value of $R^{2}$ to 1 , the better the regression performance of the GW-RF will be.

Variable importance can sort the independent (predictor) variables according to their degree of correlation to the dependent (response) variable. There are two popular methods to measure the variable importance in the RF, which are average impurity reduction (Gini importance) and mean square error (MSE) reduction. Because the result of variable importance by impurity reduction is biased (Strobl et al. 2007), many researchers have verified and suggested choosing the MSE reduction method when permuting the variables (Strobl et al. 2008; Ishwaran 2007). The MSE reduction method uses the MSE value of the out-of-bag (OOB) data to evaluate the variable importance (Cai et al. 2018). It is determined as follows:

1. Calculate the MSE of the OBB data for each tree. For tree $t$, the MSE of OOB data is calculated by Eq. (5):

$M S E_{t}=\frac{1}{N_{t}} \sum_{i=1}^{N_{t}}\left(y_{i}-\widehat{y}_{i, t}\right)^{2}$

where $N_{t}$ is the number of samples from the OBB data in the tree $t ; \widehat{y}_{i, t}$ is the prediction for the $i$ th sample of the tree $t$. 
Table 1 Definitions of indicators and sources

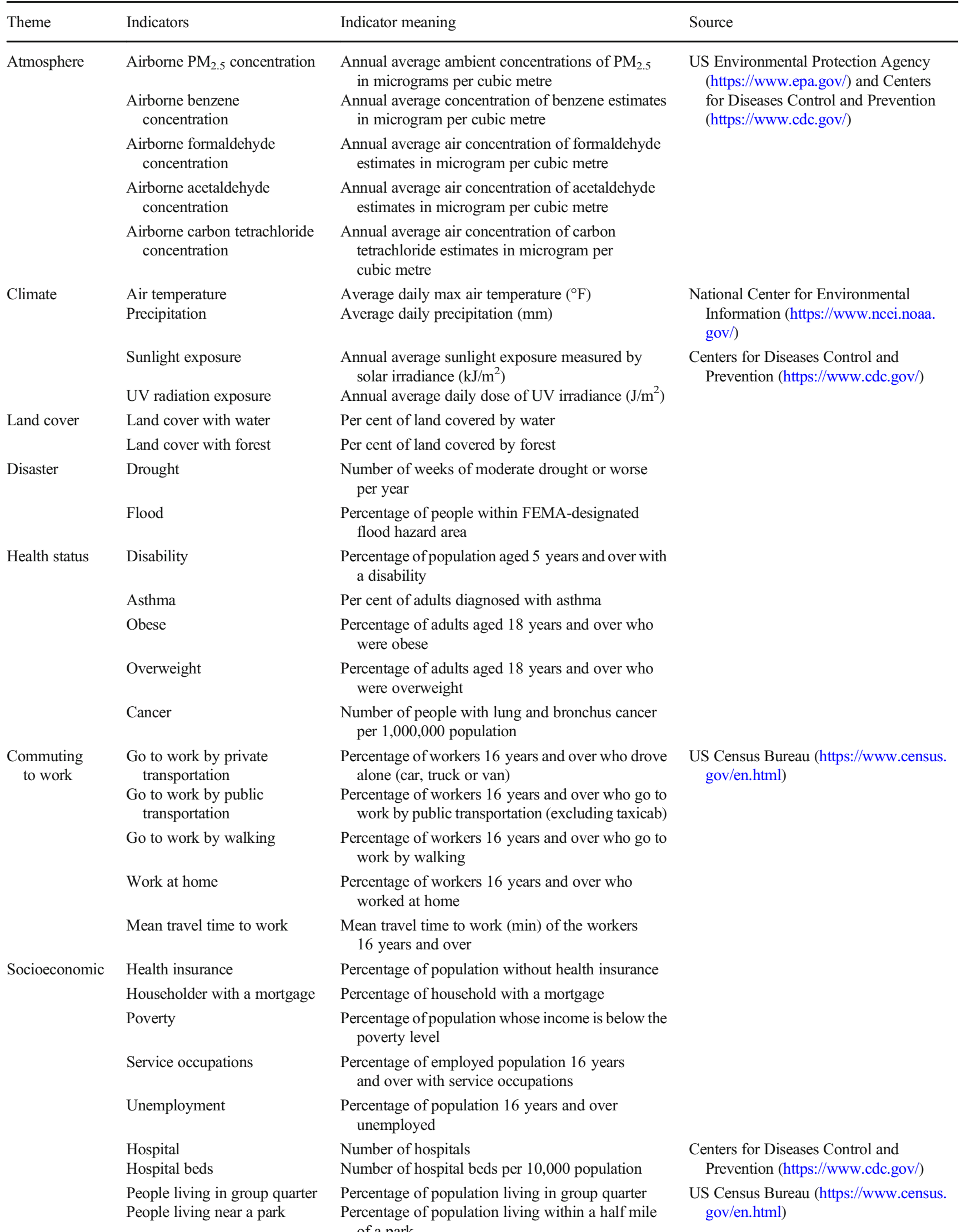

Snvironmental Protection Agency (https://www.epa.gov/) and Centers for Diseases Control and Prevention

ational Center for Environmenta Information (https://www.ncei.noaa.

Conters for Diseases Control and solar irradiance $\left(\mathrm{kJ} / \mathrm{m}^{2}\right)$

Annual average daily dose of UV irradiance $\left(\mathrm{J} / \mathrm{m}^{2}\right)$

Per cent of land covered by water

cent of land covered by forest per year

ercentage of people within FEMA-designated ercentage of population aged 5 years and over with a disability ercentage of adults aged 18 years and over who umber of people with lung and bronchus cancer per 1,000,000 population

alone (car, truck or van)

Percentage of workers 16 years and over who go to public transportation (excluding taxicab)

work by walking

ercentage of workers 16 years and over who ean travel time to work (min) of the workers 16 years and ove

Percentage of household with a mortgage

ercentage of population whose income is below the

centage of employed population 16 years unemployed

moer of hospital

Percentage of population living in group quarter of a park
S Census Bureau (https://www.census. 
Table 1 (continued)

\begin{tabular}{|c|c|c|c|}
\hline Theme & Indicators & Indicator meaning & Source \\
\hline Demographic & $\begin{array}{l}\text { Householder with no internet } \\
\text { access } \\
\text { Median household income } \\
\text { Mean household retirement } \\
\text { income } \\
\text { Mean household cash public } \\
\text { assistance income } \\
\text { Mean household supplemental } \\
\text { security income } \\
\text { Per cent of males } \\
\text { Median age } \\
\text { Per cent of people under } \\
18 \text { years } \\
\text { Per cent of people } 65 \text { years } \\
\text { and over } \\
\text { Per cent of the white race } \\
\text { Per cent of the black or } \\
\text { African American } \\
\text { Per cent of American Indian } \\
\text { and Alaska Native } \\
\text { Per cent of Asian } \\
\text { Per cent of native Hawaiian } \\
\text { and other Pacific islander } \\
\text { Per cent of Hispanic or Latino }\end{array}$ & Percentage of households with no internet access & \\
\hline
\end{tabular}

2. Randomly replace the target variable $j$, and then the new value of the MSE of tree $t$ is calculated by Eq. (6):

$\operatorname{MSE}_{t}(j)=\frac{1}{N_{t}} \sum_{i=1}^{N_{t}}\left(y_{i}-\widehat{y}_{i, t}(j)\right)^{2}$

where $\widehat{y}_{i, t}(j)$ is the prediction for the $i$ th sample of the new tree $t$ when randomly replacing the target variable $j$.

3. Calculate the difference between $M S E_{t}$ and $M S E_{t}(j)$, and the MSE reduction is the variable importance for variable $j$ of tree $t$. The MSE reduction of variable $j$ of the whole forest is obtained as the average over MSE reduction of all $n$ trees. The variable importance of variable $j$ is expressed as in Eq. (7):

$\mathrm{VI}(j)=\operatorname{MSE}(j)=\frac{1}{n} \sum_{t=1}^{n}\left(M S E_{t}-\operatorname{MSE}_{t}(j)\right)$

\section{GW-RF}

In this section, a local nonlinear machine learning method, denoted as GW-RF, is proposed. The GW-RF is designed by integrating spatial weight matrix (SWM) and RF into a local regression analysis framework. The GW-RF inherits the merits of the RF, making the RF from being applicable from a global system to a local system. Thus, it can handle high-dimensional variables with nonlinear relationships and multicollinearity. The variable importance for each spatial unit can be obtained from the GW-RF. The process of constructing the GW-RF model is designed as follows:

1. The SWM for each spatial unit of the study area should first be made according to the specified spatial weight rule. The SWM for the whole study area with $p$ spatial units can be expressed as in Eq. (8):

$W=\left[\begin{array}{c}W(1) \\ W(2) \\ \vdots \\ W(i) \\ \vdots \\ W(p)\end{array}\right]=\left[\begin{array}{cccc}w_{11} w_{12} & \cdots & w_{1 p} \\ w_{21} w_{22} & \cdots & w_{2 p} \\ \vdots & \vdots & \vdots & \vdots \\ w_{i 1} w_{i 2} & \cdots & w_{i p} \\ \vdots & \vdots & \vdots & \vdots \\ w_{p 1} w_{p 2} & \cdots & w_{p p}\end{array}\right], \quad i \in(1,2, \cdots, p)$

As the local random forest of an individual unit needs to consider the unit itself, the value of $w_{i i}$ is set to $1\left(w_{i i}=1\right)$. According to the spatial weight rule, for spatial unit $i$, if sample $j(j \in(1,2, \cdots, p) \wedge i \neq j)$ is a "neighbour" of unit $i$, 


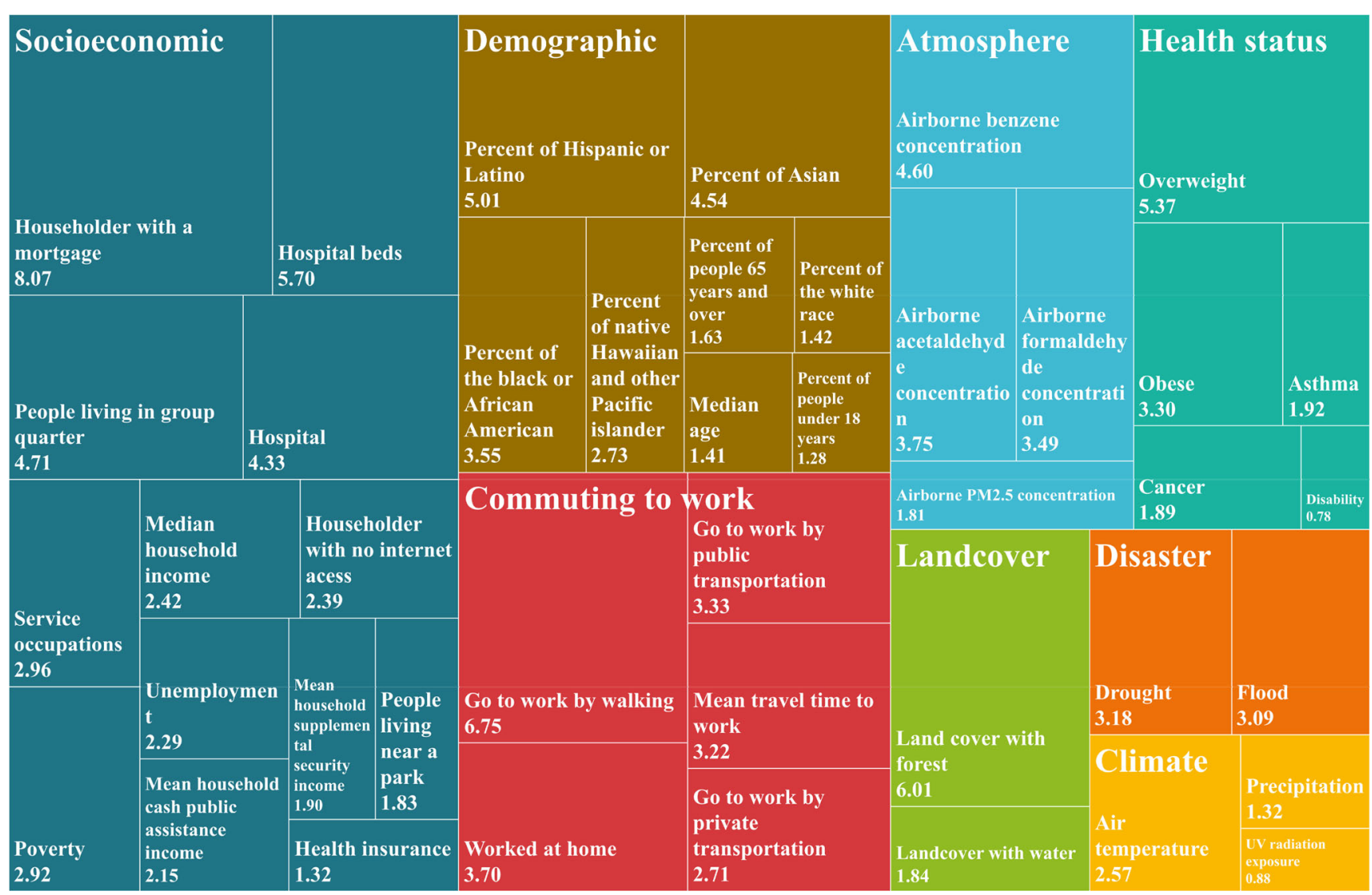

Fig. 1 The variable importance of the independent variables of the RF model in modelling COVID-19 death rate

the value of spatial weight between them is set to 1 , that is, $w_{i j}=1$. While spatial unit $j$ is far away from spatial unit $i$, not a neighbour of spatial unit $i, w_{i j}=0$.

2. Select all the neighbours of each spatial unit according to the spatial weight matrix. For unit $i$, the neighbours of it can be selected from the special weight matrix $W$ where $w_{i j} \neq 0,(j \in(1,2, \cdots, p) \wedge i \neq j)$.

3. The spatial unit $i$ and its neighbours are as the inputs to construct a local RF for unit $i$ (RF $(i)$ ). By executing RF $(i)$, the variable importance for spatial unit $i$ can be computed.

4. Repeat steps (2) and (3) to construct a local RF for each spatial unit in the study area and estimate the local variable importance for each spatial unit.

Table 2 The statistic of local $R^{2}$ of the GW-RF in modelling COVID-19 death rate; we calculated the average value of local $R^{2}$ and the percentage of counties in five local $R^{2}$ range $(\leq 0.2,(0.2,04]$, $(0.4,06],(0.6,08],>0.8)$

\begin{tabular}{ll}
\hline The value of local $R^{2}$ & GW-RF \\
\hline Average value & 0.59 \\
$\leq 0.2$ & $1.1 \%$ \\
$(0.2,04]$ & $9.5 \%$ \\
$(0.4,06]$ & $38.9 \%$ \\
$(0.6,08]$ & $44.8 \%$ \\
$>0.8$ & $5.7 \%$
\end{tabular}

The nonlinear nonparametric models (RF, GW-RF) do not need to consider multicollinearity and can analyse all independent variables without screening. R software (version 3.5.3, http://cran.r-project.org) was used to perform the regression analysis.

\section{Results}

All 47 indicators were employed to the nonlinear nonparametric models (RF, GW-RF). The adjusted fitting coefficient $\left(R^{2}\right)$ of the RF was 0.69 , while the adjusted $R^{2}$ of the GW-RF was 0.78 , indicating that the regression result of the GW-RF was more accurate than that of the RF. The variable importance of an independent variable represents the correlation between the independent variable and the dependent variable, and the higher the value of the variable importance is, the stronger the correlation will be. The variable importance of 47 independent variables in modelling COVID-19 death rate using the RF is shown in Fig. 1. The risk factors referring to socioeconomic are most correlated with COVID-19 death rate, followed by risk factors referring to demographic, commuting to work, atmosphere, health status, land cover, disaster and climate. The variables including householder with a mortgage, going to work by walking, land cover with forest, hospital beds, overweight, per cent of Hispanic or 
Fig. 2 The distribution of local $R^{2}$ of the GW-RF

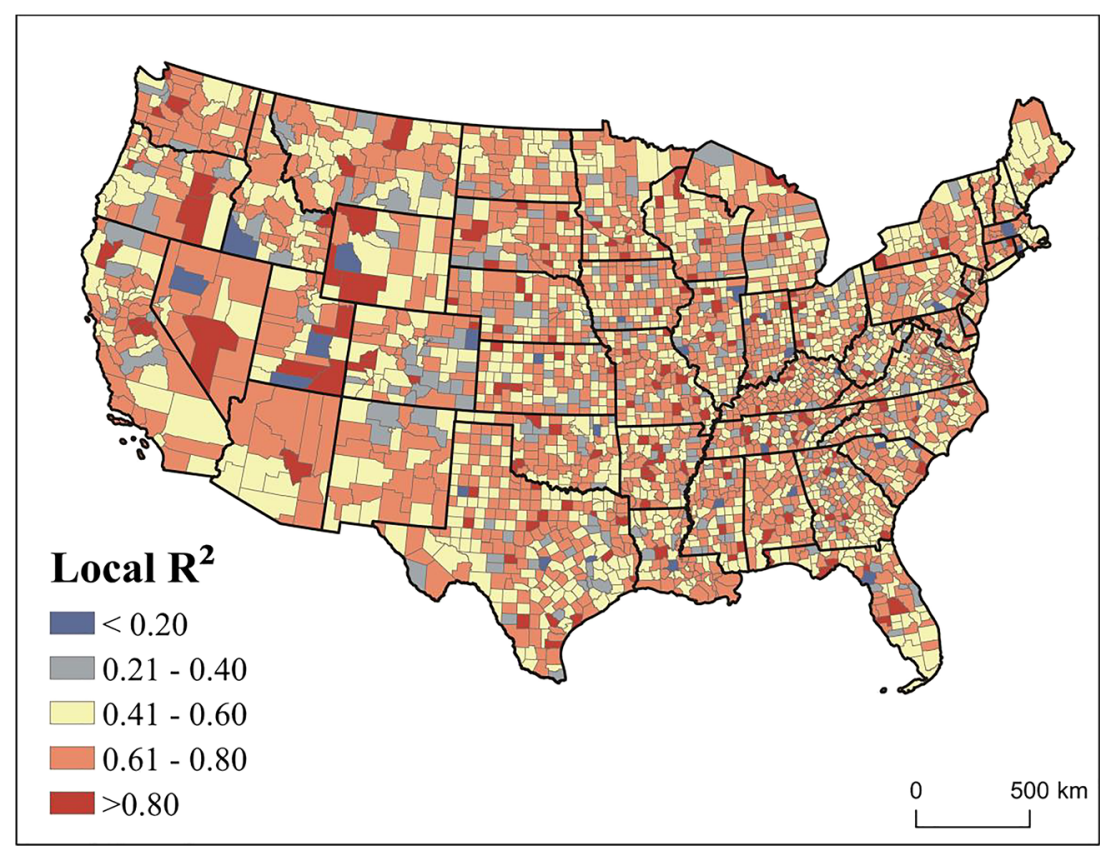

Latino, people living in group quarter and airborne benzene concentration have a high correlation with the COVID-19 death rate.

We used the local $R^{2}$ to estimate the performance of the GW-RF. Table 2 describes the statistic of local $R^{2}$ of the GWRF. The average value of local $R^{2}$ was 0.59 . The value of local $R^{2}$ was higher than 0.4 in $89.4 \%$ of the counties and higher than 0.6 in $50.5 \%$ of the counties. This shows that the GW-RF can accurately evaluate the correlation between the risk factors and the COVID-19 death rate in most of the study areas.

Figure 2 shows the distribution of the local $R^{2}$ of the GWRF across the study area. As can be seen from Fig. 2, the distribution of local $R^{2}$ was imbalanced in the whole study area. The local $R^{2}$ value of the GW-RF was high in most of the counties across the whole continental USA, indicating that the GW-RF worked well in the prediction of the local COVID-19 death rate in most regions across the study area, especially in Nevada, Arizona, Washington and some counties in the East-central region.

We computed the average local effect of each independent variable on COVID-19 death rate in the GW-RF model (see Fig. 3). The effect of going to work by walking had the highest correlation with the COVID-19 death rate, followed by airborne benzene concentration, householder with a mortgage, unemployment, airborne $\mathrm{PM}_{2.5}$ concentration and per cent of the black or African American.

The proportion of counties with local primary risk factor (the risk factor with the highest value of local variable importance) at county level in the GW-RF was calculated (see Table 3). Going to work by walking was the most influential risk factor in $35 \%$ of the counties. As SARS-CoV-2 can spread through the air, going to work by walking will shorten the social distance between people, thereby increasing the likelihood of person-to-person contact, which increases the risk of COVID-19 infection. The airborne benzene concentration was the leading risk factor in $24 \%$ of the counties. It is because that the virus always attaches to suspended particles to spread in the air, so the higher the concentration of pollution particles, the more conducive to the spread of the virus. The COVID-19 outbreak has also changed people's emotions dramatically, especially for those who are already in danger, such as people who suffer from depression. Thirteen per cent of counties were most affected by householder with a mortgage. The outbreak of COVID-19 placed great financial and emotional pressure on householders with a mortgage, which has led to them suffering from psychological illness and do not have enough money for treatment for COVID-19, thus leading to an increased risk of COVID-19. Twelve per cent of counties were most affected by unemployment. During the period of COVID-19, the unemployment rate increased greatly, and some unemployed people are more inclined to have negative emotions, which in turn are more likely to suffer from depression. Moreover, depression is not conducive to the treatment of COVID-19 patients, thus leading to an increased COVID19 death rate. Figures 4,5 and 6 provide a detailed spatial distribution of the local variable importance of the first six factors with the highest value of average variable importance on the COVID-19 death rate using the GW-RF.

From Figs. 4, 5 and 6, the distribution of the variable importance of each variable on COVID-19 death rate in GW-RF model was imbalanced in different counties even the counties in the same state. For example, in the southern part of Arizona, the COVID-19 death rate was mainly affected by the airborne benzene concentration and unemployment, and the northern part was mainly affected by going to work by walking and airborne $\mathrm{PM}_{2.5}$ 


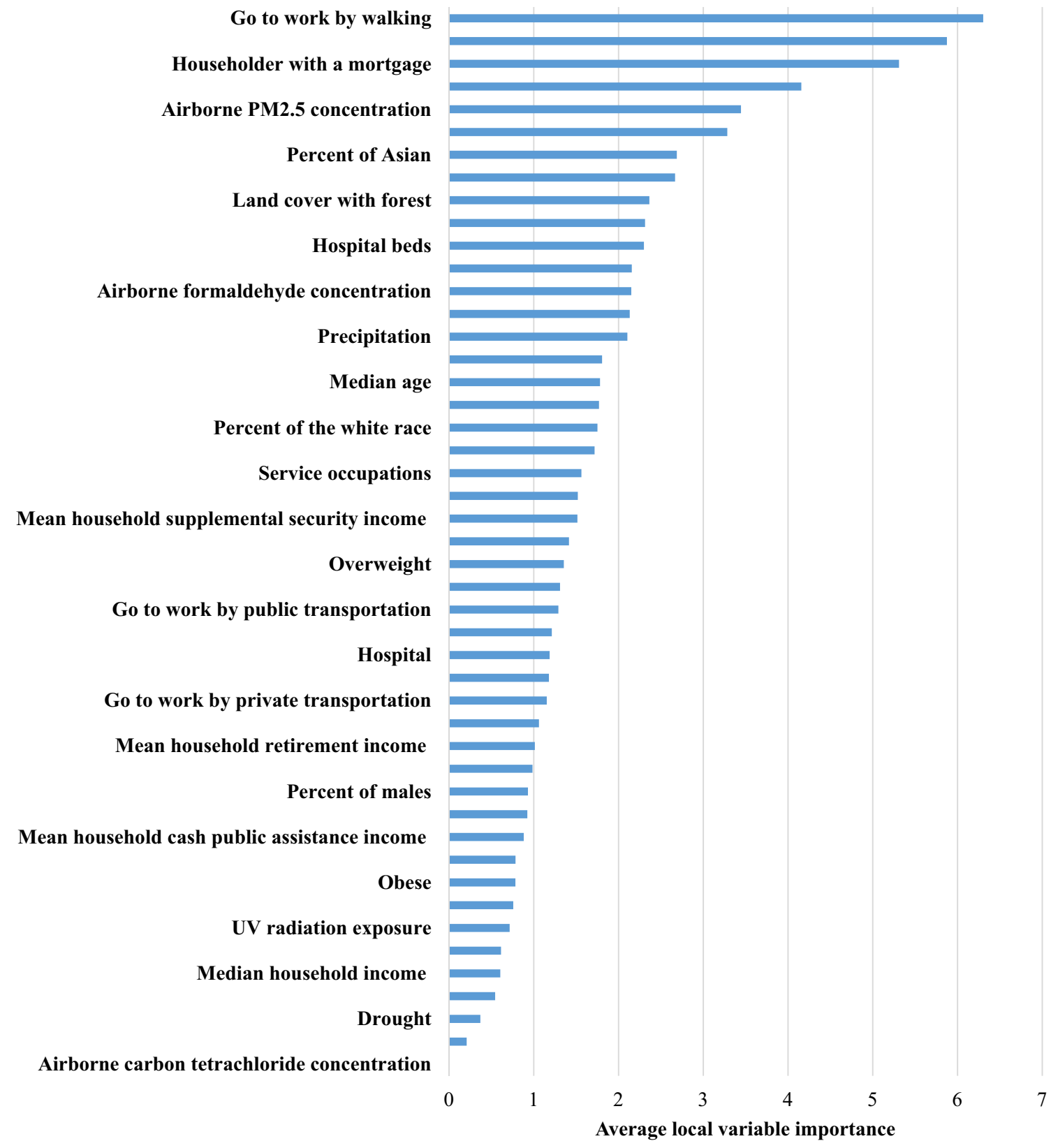

Fig. 3 The average local variable importance of 47 potential risk factors on COVID-19 death rate in the GW-RF model

concentration. The regions obviously affected by going to work by walking were distributed in California, Arizona, the west of

Table 3 The proportion of counties with local primary risk factor (the risk factor with the highest value of local variable importance) on COVID-19 death rate at county level in the GW-RF

\begin{tabular}{ll}
\hline Local primary risk factor & Proportion of counties \\
\hline Go to work by walking & $35 \%$ \\
Airborne benzene concentration & $25 \%$ \\
Householder with a mortgage & $13 \%$ \\
Unemployment & $12 \%$ \\
Other risk factors & $16 \%$ \\
\hline
\end{tabular}

Utah, South Carolina and Massachusetts. The areas influenced by airborne benzene concentration were scattered throughout the study area. New Mexico, Florida, Texas, Missouri, the south of Nevada, the north of Arizona, Massachusetts and Connecticut were sensitive to householder with a mortgage. The regions obviously affected by airborne $\mathrm{PM}_{2.5}$ concentration and per cent of the black or African American are similar, mainly located in the north of Nevada, the north of Arizona, the southeast of Oregon, the east of Wyoming and the central part of the continental USA. In addition, the same area was affected by several risk factors. For example, airborne benzene concentration, householder with a mortgage, unemployment and the per cent of black of African American were influential factors in the southeast of Arizona. 
Fig. 4 The spatial distribution of the local variable importance of a going to work by walking and $\mathbf{b}$ airborne benzene concentration on COVID-19 death rate in GWRF model
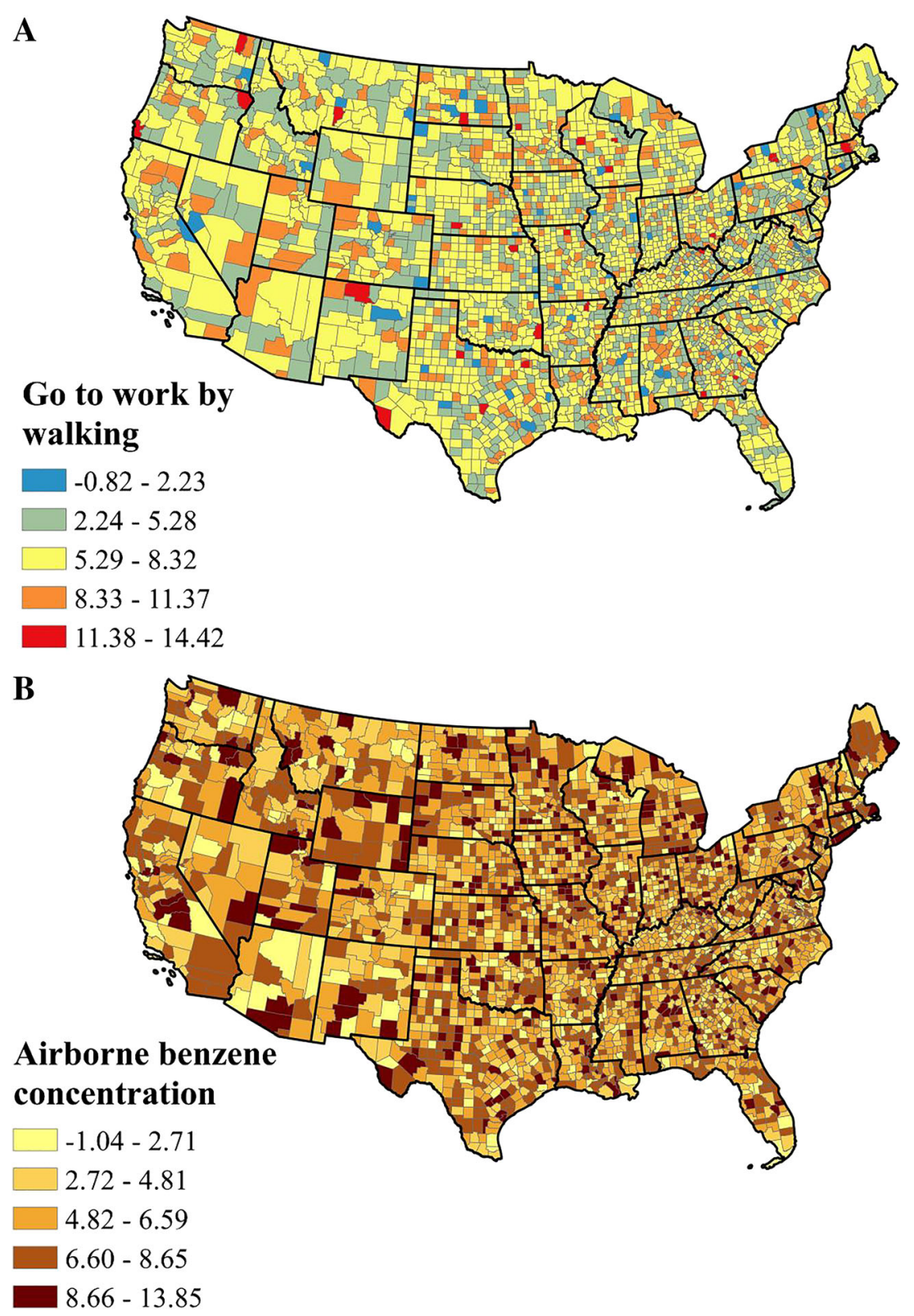

\section{Discussion and conclusion}

Identifying the risk factors that highly correlated with the transmission will provide guidance in containing the spread of the COVID-19 disease. In this study, we selected 47 potential risk factors from atmosphere, climate, land cover, disaster, health status, commuting to work and socioeconomic and demographic categories as independent variables to estimate their impact on the distribution of the COVID-19 death rate at county level across continental USA. Due to the imbalanced distribution of COVID-19 death rate and the complex relationship between the COVID-19 death rate and its risk factors, the linear models could not accurately identify the key risk factors in different locations. To solve this problem, we applied GW$\mathrm{RF}$, a local regression model capable of identifying nonlinear relationships between variables at various geographical locations and suitable for dealing with high-dimensional variables even for correlated variables.

In this study, we used two nonlinear regression models (RF, GW-RF) to identify the key risk factors to the COVID19 death rate. The result showed that the nonlinear models effectively modelled the relationship between the risk factors and the COVID-19 death rate both in global and local regressions. The adjusted $R^{2}$ of the GW-RF was 0.78 , higher than that of the RF, indicating the GW-RF is more suitable to estimate the local risk factors of the COVID-19 death rate compared with the global model RF. The average value of local $R^{2}$ of the GW-RF is 0.59 . In GW-RF, the value of local $R^{2}$ is higher than 0.4 in $89.4 \%$ of the counties and higher than 0.6 in $50.5 \%$ of the counties, indicating that the GW-RF 
Fig. 5 The spatial distribution of the local variable importance of a householder with a mortgage and b unemployment on COVID-19 death rate in GW-RF model
A

\section{Householder with a mortgage}

$-0.64-3.23$

$3.24-4.68$

$4.69-5.95$

$5.96-7.41$

$7.42-11.58$

B

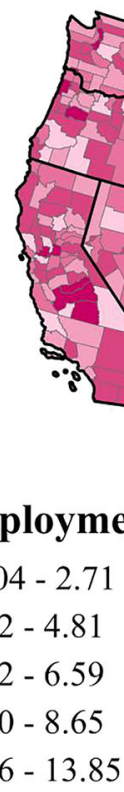

performed well in most of the study area. This shows that that the local nonlinear nonparametric model GW-RF can accurately estimate the relationship between the risk factors and COVID-19 death rate at various geographical locations.

Our result shows that several risk factors from environment, socioeconomic, demographic and commuting to work are associated with the COVID-19 death rate. Finding of the global model RF showed that householders with a mortgage had the highest correlation with the number of COVID-19 death rate, followed by going to work by walking, land cover with forest, hospital beds and overweight. Findings of the geographical local model GW-RF is similar to that of the RF, but a little different. The GW-RF results show that going to work by walking, airborne benzene concentration, householder with a mortgage, unemployment, airborne $\mathrm{PM}_{2.5}$ concentration and per cent of the black or
African American played an important role in the distribution of the COVID-19 death rate. Most of our findings are consistent with previous research on COVID-19. Zheng et al. (2020) found that the frequency of public transportation including flights, trains and buses from the epicentre is an important determinant of transmission risks of COVID-19. They suggested preventive measures should be taken in public transportation in order to contain the COVID-19 epidemic. Several studies found that air pollution has a significant correlation with the COVID-19 confirmed cases (Xu et al. 2020; Bashir et al. 2020). Viruses are usually not spread as independent individuals in air; they are more likely to attach to other suspended particles (Yang et al. 2011). Therefore, the concentration of air pollutants may affect the aerosol transmission of SARS-CoV-2. These studies encouraged the formulation of environmental policies to control 
Fig. 6 The spatial distribution of the local variable importance of a airborne $\mathrm{PM}_{2.5}$ concentration and b per cent of the black or African American on COVID-19 death rate in GW-RF model
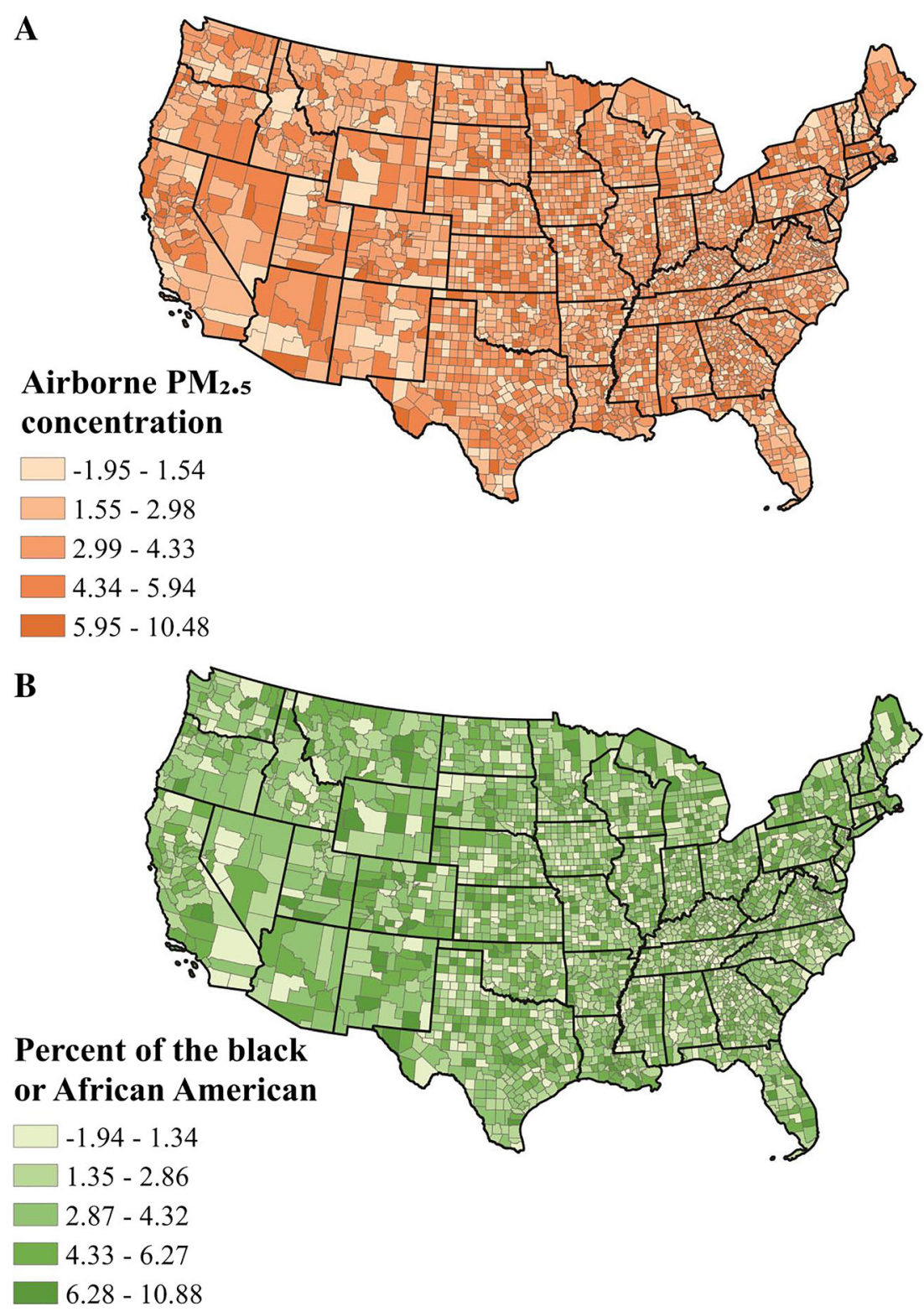

pollution sources, which can reduce the harmful effects of air pollutants. Studies from Li et al. (2020) and DiMaggio et al. (2020) showed that compared with the general population of the USA, black Americans were at apparently higher risk of COVID-19 infection and mortality nationwide. It is probably because black Americans suffer more from poverty, environmental pollution, overcrowded housing and less access to health care than do the general population of the USA. The prevalence of smoking and chronic diseases such as cardiovascular disease, diabetes, hypertension, obesity and chronic respiratory diseases has increased among black Americans, all of which increase the risk of COVID-19 (Fang et al. 2020; Zhou et al. 2020; Fouad et al. 2020). Mollalo et al. (2020) found that the proportion of black females and median household income had significant influence on the spatial distribution of the COVID-19 incidence rate.
By exploring the spatial distribution of risk factors of the COVID-19 death rate, we found that COVID-19 death rate in each region was affected by various factors, and the association between each risk factor and the COVID-19 death rate was not consistent in different spatial locations. The result showed that going to work by walking, airborne benzene concentration, householder with a mortgage, unemployment, airborne $\mathrm{PM}_{2.5}$ concentration and per cent of the black or African American had significant relation with the distribution of the COVID-19 death rate. Other risk factors such as mean travel time to work, hospital distribution and air temperature may require more data to estimate their relationship with the distribution of the COVID-19 death rate. About $35 \%$ of the counties are most affected by going to work by walking, so it is necessary to call on people to pay attention to social distancing and to wear medical masks. The western and 
central east regions were affected by the airborne benzene concentration; toxic particles in the air affect the spread of viruses. Therefore, these regions should pay attention to the impact of air pollution on human health and take measures to protect the environment. The southern part of the continental USA was heavily affected by the proportion of the black or African American and householder with a mortgage, so some assistance probably can be taken in these regions to provide people with financial help such as food and medical supplies.

The current research, despite showing the spatial variability of the correlation between multiple risk factors and the COVID-19 death rate at a county level, has the following limitations. First, the current study only focused on the spatial dimension of the data based on a period, but the data about the COVID-19 death rate is constantly changing over time. Future study can study its spatiotemporal distribution. Secondly, we do not account for policy factors at local area. Policy factors would be an interesting research contribution to the transmission of COVID-19. Thirdly, the GW-RF model only assesses the goodness-of-fit test of the regression but does not assess the significance of the single variable. The test method of this model needs to be improved in the future study.

At present, few geographic local models study the nonlinear relationship between variables. The proposed GWRF model could accurately estimate the spatial variability of nonlinear relationship between the risk factors and COVID-19 death rate; thus, this method is applicable in many use instances where this is an issue about selecting significantly correlated variables at various geographical locations. Our results confirmed the findings of existing work on COVID-19 but extend it by using a nonlinear approach to quantify the impact of risk factors relevant in local areas. We expect this study could provide a reference for the geographical local nonlinear modelling in the future epidemiological studies.

Author contribution Yaowen Luo: conceptualization, writing - original draft, formal analysis, methodology, software, formal analysis, investigation, visualization. Jianguo Yan: conceptualization, validation, writingreview and editing, supervision, funding acquisition. Stephen McClure: validation, writing - review and editing.

Funding This research is supported by a grant provided by National Scientific Foundation of China (Grant No. U1831132 and 41374024), Innovation Group of Natural Fund of Hubei province (Grant No. 2018CFA087) and the fundamental research funds for the central universities (2042018KF0231).

Data availability The datasets used during the current study are available from the corresponding author on reasonable request.

\section{Compliance with ethical standards}

Competing interests The authors declare that they have no competing interests.
Ethical approval Not applicable.

Consent for publication All the co-authors consent the publication of this work.

Consent to participate Not applicable.

\section{References}

Ahmadi et al (2020) Investigation of effective climatology parameters on COVID-19 outbreak in Iran. Sci Total Environ 729:138705. https:// doi.org/10.1016/j.scitotenv.2020.138705

Bashir, et al. (2020) Correlation between environmental pollution indicators and COVID-19 pandemic: a brief study in Californian context. Environ Res 109652. https://doi.org/10.1016/j.envres.2020.109652

Breiman (2001) Random forests. Machine Learning 45(1):5-32

Brunsdon et al (2010) Geographically weighted regression : a method for exploring spatial nonstationarity. Geogr Anal 28(4):281-298. https://doi.org/10.1111/J.1538-4632.1996.TB00936.X

Cai et al (2018) A synthesis of disaster resilience measurement methods and indices. Int J Dis Risk Reduct 31:844-855. https://doi.org/10. 1016/J.IJDRR.2018.07.015

Calderón-Larrañaga et al (2020) COVID-19: risk accumulation among biologically and socially vulnerable older populations. Ageing Res Rev 63:101149. https://doi.org/10.1016/j.arr.2020.101149

Dariya, Nagaraju (2020) Understanding novel COVID-19: its impact on organ failure and risk assessment for diabetic and cancer patients. Cytokine Growth Factor Rev 53:43-52. https://doi.org/10.1016/j. cytogfr.2020.05.001

de León-Martínez et al (2020) Critical review of social, environmental and health risk factors in the Mexican indigenous population and their capacity to respond to the COVID-19. Sci Total Environ 139357:139357. https://doi.org/10.1016/j.scitotenv.2020.139357

Desjardins et al (2020) Rapid surveillance of COVID-19 in the United States using a prospective space-time scan statistic: detecting and evaluating emerging clusters. Appl Geogr 118:102202. https://doi. org/10.1016/j.apgeog.2020.102202

DiMaggio et al (2020) Blacks/African American communities are at highest risk of COVID-19: spatial modeling of New York city ZIP code-level testing results. Ann Epidemiol 51:7-13. https://doi.org/ 10.1016/j.annepidem.2020.08.012

Fang et al (2020) Are patients with hypertension and diabetes mellitus at increased risk for COVID-19 infection? Lancet Respir Med 8(4): e21. https://doi.org/10.1016/S2213-2600(20)30116-8

Fotheringham et al (2003) Geographically weighted regression: the analysis of spatially varying relationships. John Wiley \& Sons, Hoboken

Fouad et al (2020) COVID-19 is disproportionately high in African Americans. This will come as no surprise... Am J Med. https:// doi.org/10.1016/j.amjmed.2020.04.008

Grömping (2009) Variable importance assessment in regression: linear regression versus random forest. Am Stat 63(4):308-319. https:// doi.org/10.1198/TAST.2009.08199

Guliyev (2020) Determining the spatial effects of COVID-19 using the spatial panel data model. Spatial Stat 38:100443. https://doi.org/10. 1016/j.spasta.2020.100443

Huang et al (2020) Clinical features of patients infected with 2019 novel coronavirus in Wuhan, China. Lancet 395(10223):497-506. https:// doi.org/10.1016/S0140-6736(20)30183-5

Imran et al (2015) Using geographically weighted regression kriging for crop yield mapping in West Africa. Int J Geogr Inf Sci 29(2):234 257. https://doi.org/10.1080/13658816.2014.959522 
Ishwaran (2007) Variable importance in binary regression trees and forests. Electronic J Stat 1:519-537. https://doi.org/10.1214/07EJS039

Landi (2020) The geriatrician: the frontline specialist in the treatment of covid-2019 patients. J Am Med Dir Assoc 21:937-938. https://doi. org/10.1016/j.jamda.2020.04.017

Lau et al (2020) The association between international and domestic air traffic and the coronavirus (COVID-19) outbreak. J Microbiol Immunol Infect 53:467-472. https://doi.org/10.1016/j.jmii.2020. 03.026

Li et al (2020) Multivariate analysis of black race and environmental temperature on COVID-19 in the US. Am J Med Sci 360:348356. https://doi.org/10.1016/j.amjms.2020.06.015

Liu et al (2020) Using the contact network model and MetropolisHastings sampling to reconstruct the COVID-19 spread on the "diamond princess". Sci Bull 65:1297-1305. https://doi.org/10.1016/j. scib.2020.04.043

$\mathrm{Lu}$ et al (2017) Geographically weighted regression with parameterspecific distance metrics. Int J Geogr Inf Sci 31(5):982-998. https://doi.org/10.1080/13658816.2016.1263731

Malik et al (2020) Higher body mass index is an important risk factor in COVID-19 patients: a systematic review and meta-analysis. Environ Sci Pollut Res. https://doi.org/10.1007/s11356-020-10132-4

Marhl et al (2020) Diabetes and metabolic syndrome as risk factors for COVID-19. Diabetes Metab Syndr Clin Res Rev 14:671-677. https://doi.org/10.1016/j.dsx.2020.05.013

Millett et al (2020) Assessing differential impacts of COVID-19 on black communities. Ann Epidemiol 47:37-44. https://doi.org/10.1016/j. annepidem.2020.05.003

Mollalo et al (2020) GIS-based spatial modeling of COVID-19 incidence rate in the continental United States. Sci Total Environ 728:138884. https://doi.org/10.1016/j.scitotenv.2020.138884

O'Connor et al (2020) Economic recovery after the COVID-19 pandemic: resuming elective orthopedic surgery and total joint arthroplasty. J Arthroplast 35:S32-S36. https://doi.org/10.1016/j.arth.2020.04. 038

Pourali et al (2020) Relationship between blood group and risk of infection and death in COVID-19: a live meta-analysis. New Microbes New Infect. https://doi.org/10.1016/J.NMNI.2020.100743

Rosenkrantz, et al. (2020) The need for GIScience in mapping COVID19. Health Place 102389. doi https://doi.org/10.1016/j.healthplace. 2020.102389

Ruthberg et al (2020) Geospatial analysis of COVID-19 and otolaryngologists above age 60. Am J Otolaryngol 102514:102514. https://doi. org/10.1016/j.amjoto.2020.102514

Sannigrahi et al (2020) Examining the association between sociodemographic composition and COVID-19 fatalities in the European region using spatial regression approach. Sustain Cities Soc 62:102418. https://doi.org/10.1016/j.scs.2020.102418

Serge et al (2020) Are we equal in adversity? Does Covid-19 affect women and men differently? Maturitas. 138:62-68. https://doi.org/ 10.1016/j.maturitas.2020.05.009
Shim et al (2020) Transmission potential and severity of COVID-19 in South Korea. Int J Infect Dis 93:339-344. https://doi.org/10.1016/j. ijid.2020.03.031

Strobl et al (2007) Bias in random forest variable importance measures: illustrations, sources and a solution. BMC Bioinf 8(1):25. https:// doi.org/10.1186/1471-2105-8-25

Strobl et al (2008) Conditional Variable Importance for Random Forests. BMC Bioinf 9(1):307. https://doi.org/10.1186/1471-2105-9-307

Sun et al (2020) A spatial analysis of COVID-19 period prevalence in US counties through June 28, 2020: where geography matters? Ann Epidemiol. https://doi.org/10.1016/j.annepidem.2020.07.014

Targher et al (2020) Patients with diabetes are at higher risk for severe illness from COVID-19. Diabetes Metab 46:335-337. https://doi. org/10.1016/j.diabet.2020.05.001

Tosepu et al (2020) Correlation between weather and Covid-19 pandemic in Jakarta, Indonesia. Sci Total Environ 725:138436. https://doi.org/ 10.1016/j.scitotenv.2020.138436

Wang et al (2020) Immediate psychological responses and associated factors during the initial stage of the 2019 coronavirus disease (COVID-19) epidemic among the general population in China. Int J Environ Res Public Health 17(5). https://doi.org/10.3390/ IJERPH17051729

Wheeler, Tiefelsdorf (2005) Multicollinearity and correlation among local regression coefficients in geographically weighted regression. J Geogr Syst 7(2):161-187. https://doi.org/10.1007/S10109-0050155-6

World Health Organization (2020) Coronavirus disease (COVID-19) outbreak situation [online]. https://www.who.int/emergencies/ diseases/novel-coronavirus-2019. [Accessed 21 March 2020]

$\mathrm{Xu}$ et al (2020) Possible environmental effects on the spread of COVID19 in China. Sci Total Environ 731:139211. https://doi.org/10.1016/ j.scitotenv.2020.139211

Yang et al (2011) Concentrations and size distributions of airborne influenza A viruses measured indoors at a health centre, a day-care centre and on aeroplanes. J R Soc Interface 8(61):1176-1184. https://doi. org/10.1098/RSIF.2010.0686

Zaki et al (2020) Association of hypertension, diabetes, stroke, cancer, kidney disease, and high-cholesterol with COVID-19 disease severity and fatality: a systematic review. Diabetes Metab Syndr Clin Res Rev 14(5):1133-1142. https://doi.org/10.1016/j.dsx.2020.07.005

Zheng et al (2020) Spatial transmission of COVID-19 via public and private transportation in China. Travel Med Infect Dis 34:101626. https://doi.org/10.1016/j.tmaid.2020.101626

Zhou et al (2020) Clinical course and risk factors for mortality of adult inpatients with COVID-19 in Wuhan, China: a retrospective cohort study. Lancet 395(10229):1054-1062. https://doi.org/10.1016/ S0140-6736(20)30566-3

Publisher's note Springer Nature remains neutral with regard to jurisdictional claims in published maps and institutional affiliations. 